\title{
Applications of simulated microgravity conditions in Space microbiology
}

\author{
Yesupatham Sathish Kumar, Sunirmal Sheet and Yang Soo Lee* \\ Department of Forest Science and Technology, Chonbuk National University, South Korea
}

Submission: March 29, 2017; Published: March 31, 2017

*Corresponding author: Yang Soo Lee, Department of Forest Science and Technology, College of Agriculture and Life Sciences, Chonbuk National University, Jeonju 561-756, Republic of Korea, South Korea

Abbreviations: F: Force; LEO: Low Earth Orbit; RCCS: Rotatory Cell Culture Systems; RPM: Random Positioning Machine; FFM: Free Fall Machine; CFFM: Centrifuge Free Fall Machine; HARV: High Aspect Ratio Vessel

\section{Mini Review}

Gravity is well-known, yet little understood physical force. Since life ever appeared, its intensity and direction have been a constant selection force throughout the evolutionary history of the Earth. The ubiquitous presence of gravitational force makes it difficult to understand its role in life if any. Gravity is one of the multiple physical factors determining the behavior and development of all organisms. Gravity influences humans directly. The gravitational force is $10^{38}$ times weaker than the nuclear force. However, gravitational force has infinite range, positively acted on all particles with mass/energy. An object with a mass $(\mathrm{m})$ on the surface of the Earth accelerates towards the Earth's Centre at approximately $9.81 \mathrm{~ms}^{-2}$. This acceleration due to gravity on the Earth's surface is generally considered as 1g (one Earth gravity). Gravity ideally can impose two effects on an object as a function of its mass: displacement (motion) and/or deformation (weight). As long as gravity is present, either one or both of these reactions will occur and can be analyzed primarily as follows. The gravitational constant $(\mathrm{G}=$ $6.672 \times 10-11 \mathrm{~N} \bullet \mathrm{m}^{2} \bullet \mathrm{kg}^{-2}$ ) is neither a force nor an acceleration intrinsically, but a physical constant used to dimensionally derive the force $\left(\mathrm{F}_{1,2}\right)$ resulting from the attraction by a particle of mass $\left(m_{1}\right)$ on another mass particle $\left(m_{2}\right)$ at a distance $(r)$. The magnitude of this attractive force is determined by Newton's law of gravitation, $F_{1,2}=\left(G m_{1} m_{2}\right) r^{-2}$.

The equation of force $(F)$ governing weight is derived from taking into account the gravitational acceleration (a) at the surface of the Earth $\left(9.81 \mathrm{~m} \bullet \mathrm{s}^{-2}\right.$ or $\left.1 \times \mathrm{g}\right)$ acting on a given mass $(m): F=m a$. The outcome of this relationship is so ubiquitous in our daily lives that weight is usually not considered a variable to be manipulated experimentally. Even in low Earth orbit, the force of gravity is not actually removed. Rather, without the significant equal and opposite resistance needed to impart weight, an orbiting object is simply experiencing a continuous state of gravity-induced free fall (i.e., accelerated motion) around the Earth. This state is what is commonly referred to as "weightlessness" or "microgravity," as attributed to a relative frame of reference in which an object appears to "float" inside the spacecraft.

Our knowledge on the effects of microgravity on cells, microbes, plants and animals are derived from a comparison of experiments conducted in space vehicles with ground controls in $1 \mathrm{~g}$. On-board centrifuges in space vehicles can provide $\mathrm{g}$-forces from near zero to more than $1 \mathrm{~g}$ to be used as controls in microgravity environments. An actual microgravity environment can be provided for a short duration by generating free fall conditions close to the Earth's surface with parabolic flights and drop facilities. Space vehicles are unique biological laboratories. Space biology experiments are generally carried out in low Earth orbit (LEO), at altitudes less than $1 \%$ of the distance from the Earth to the Moon. Some of the familiar LEO vehicles are Sputnik I (215 km), ISS (350 km), Mir (390 km) and space shuttles (300$400 \mathrm{~km}$ ). At a distance of about $300 \mathrm{~km}$ above the Earth, the force of gravity is still as high as $90 \%$ as on Earth. However, space vehicles moving in circular LEO experience actual microgravity environments because they are in free fall. The centripetal force towards the Earth is balanced by the centrifugal acceleration, resulting in the free fall or microgravity condition.

\section{Ground-Based Analogues of Microgravity}

Ground-Based models play a significant role in space exploration because they provide important data for the design of space flight experiments. Experiments performed using simulators can be scheduled without trouble for crew time, and modifications could be made with trivial impact on cost. The 
model should be well defined by its limitations and the results obtained should mimic the data obtained with the space flight studies before it is deemed as a ground-based model. One such model is the Hind limb unloading rodent model used in the mid1970. The rodent's hind limb is elevated to produce a $30^{\circ}$ tilt in the head, which results in a cephalic fluid shift and avoids weight bearing by the hindquarters. This model demanded skill, experience and also the mice cannot be used for long-term experiments. The need for alternative models arose which lead to the invention of the Rotatory Cell Culture Systems (RCCS) by the NASA Biotechnology division based at the Johnson space center, Texas.

The RCCS is a powerful laboratory tool with simple concepts and innovation. Several modified versions of the apparatus are on the way. The RCCS is essentially a standardized form of suspension culture and consists of a hollow disk or cylinder that is completely filled with medium (no bubbles, i.e., "zero headspace") and rotates on an axis parallel to the ground (and perpendicular to the gravitational force vector. There are many other approaches to simulate the microgravity by randomizing the gravitational vector like a Random Positioning Machine (RPM), Free Fall Machine (FFM) and Centrifuge Free Fall Machine (CFFM). Levitation by using diamagnetic properties of the materials a new method has been devised using high magnetic field for creating a state of "magnetic microgravity". High Aspect ratio vessel (HARV) is one of the variants of RCCS, our research group has adopted HARV to obtain a low shear force on the fungal cell wall, simulating "microgravity" conditions and documented various morphological, physiological and genomic changes in a few filamentous fungal species.

\section{Conclusion}

The role that gravity plays in biological processes can be revealed explicitly under its absence/microgravity condition. The force of gravity has been relatively constant for the duration of the evolutionary processes of life on Earth. Our venture into Space and low gravity environments like spaceships, demands the study on the adaptive processes occurring in the cells under microgravity condition. The mounting necessity resulted in the invention of various ground- based analogues, simulating microgravity conditions on earth within the lab setup. Based on the simulated microgravity experiments, our understanding of the role of gravity in shaping the evolution on Earth will eventually step-up. Investigations in lower species like Bacteria, Fungi with short generation times may reveal the underlying mode of survival and eventual thriving of living organisms under microgravity.

\section{Your next submission with Juniper Publishers will reach you the below assets}

- Quality Editorial service

- Swift Peer Review

- Reprints availability

- E-prints Service

- Manuscript Podcast for convenient understanding

- Global attainment for your research

- Manuscript accessibility in different formats

( Pdf, E-pub, Full Text, Audio)

- Unceasing customer service

Track the below URL for one-step submission https://juniperpublishers.com/online-submission.php 